University of Nebraska - Lincoln

DigitalCommons@University of Nebraska - Lincoln

Roger Kirby Publications

Research Papers in Physics and Astronomy

February 1976

\title{
Raman spectrum of gadolinium molybdate at $80^{\circ} \mathrm{K}$
}

\author{
B.N. Ganguly \\ University of Nebraska-Lincoln \\ Frank G. Ullman \\ University of Nebraska-Lincoln \\ Roger D. Kirby \\ University of Nebraska-Lincoln, rkirby1@unl.edu \\ John R. Hardy \\ University of Nebraska-Lincoln
}

Follow this and additional works at: https://digitalcommons.unl.edu/physics_kirby

Part of the Physics Commons

Ganguly, B.N. ; Ullman, Frank G.; Kirby, Roger D.; and Hardy, John R., "Raman spectrum of gadolinium molybdate at 80" (1976). Roger Kirby Publications. 27.

https://digitalcommons.unl.edu/physics_kirby/27

This Article is brought to you for free and open access by the Research Papers in Physics and Astronomy at DigitalCommons@University of Nebraska - Lincoln. It has been accepted for inclusion in Roger Kirby Publications by an authorized administrator of DigitalCommons@University of Nebraska - Lincoln. 


\title{
Raman spectrum of gadolinium molybdate at $80^{\circ} \mathrm{K} \dagger$
}

\author{
B. N. Ganguly, ${ }^{\ddagger}$ Frank G. Ullman, R. D. Kirby, and J. R. Hardy \\ Behlen Laboratory of Physics, University of Nebraska, Lincoln, Nebraska 68508
}

(Received 9 June 1975)

\begin{abstract}
Lines that overlap from thermal broadening in the room-temperature Raman spectrum of gadolinium molybdate are resolved in the $80^{\circ} \mathrm{K}$ spectrum. A total of 126 of the possible 201 lines have been resolved. Symmetry assignments are derived from considerations of both point symmetry and polariton dispersion, supported by separate measurements of $A_{1}(\mathrm{TO})$ and $A_{1}(\mathrm{LO})$ spectra. The splitting of the unstable $47 \mathrm{~cm}^{-1}$ room-temperature $A_{1}(\mathrm{TO})$ line into a doublet at 44.5 and $51.5 \mathrm{~cm}^{-1}$, observed previously only in ir absorption, is also observed in these spectra. On heating, the $44.5-\mathrm{cm}^{-1}$ line remains fixed in frequency whereas the 51.5$\mathrm{cm}^{-1}$ line broadens and shifts toward lower frequency until, above $210^{\circ} \mathrm{K}$, the $44.5-\mathrm{cm}^{-1}$ line is obscured. Another $A_{1}$ (TO) line, peaking at $83 \mathrm{~cm}^{-1}$ at $80^{\circ} \mathrm{K}$, shifts toward lower frequency on heating, reaching 75 $\mathrm{cm}^{-1}$ at room temperature and remaining at that value on further heating up to the transition at $159^{\circ} \mathrm{C}$ where it becomes Raman inactive. The broadening of the $51.5-\mathrm{cm}^{-1}$ line in the $A_{1}(\mathrm{LO})$ spectrum is less pronounced so its correlation with the modes of the high-temperature phase can be determined unambiguously. These three lines are suggested to be associated with a system of three coupled modes, whose behavior is discussed, qualitatively, in terms of energy transfer between modes and damping via a two-phonon decay involving an optic phonon of about $44.5 \mathrm{~cm}^{-1}$ and a long-wave acoustic phonon.
\end{abstract}

\section{INTRODUCTION}

The Raman spectrum of gadolinium molybdate, $\mathrm{Gd}_{2}\left(\mathrm{MoO}_{4}\right)_{3}$, has been studied previously at room temperature and above its $159^{\circ} \mathrm{C}$ ferroelectric transition. ${ }^{1}$ of a possible 201 Raman-active modes in the ferroelectric phase, only 68 could be identified. Since many of the calculated phonon energies were found to be closely spaced ${ }^{2}$ and the anomalous, room-temperature $47-\mathrm{cm}^{-1} A_{1} \operatorname{mode} \mathrm{e}^{3,4}$ had been reported to split into a doublet at low temperature, ${ }^{5}$ it was clear that further resolution and identification would be forthcoming from measurements of the Raman spectrum at lower temperature. In this paper, the spectrum measured at $80^{\circ} \mathrm{K}$ is described and classified. In addition, the effect of heating from $80^{\circ} \mathrm{K}$ on the three lowestfrequency $A_{1}$ lines, that appear to be associated with the ferroelectric transition, is described and interpreted.

In the room-temperature spectrum, described previously, ${ }^{1}$ some lines appeared in more than one symmetry. The symmetry assignment for a given line was taken to be the one in which the line appeared with the highest intensity. The appearance of lines of the same frequency in other symmetries was thought to result from "leakage" (from misalignment and the effect of the collecting angle of the focusing lens) from the orientation in which it appeared with highest intensity. In the work described here, examination of the changes in the spectrum with small changes in crystal alignment showed clearly which lines could be attributed to leakage; in practically all cases, leakage could be ruled out as a source of the observed degeneracies. That such degeneracies exist is not surpris- ing: not only in view of the close spacing of the calculated phonon frequencies ${ }^{2}$ but also because of the nearly horizontal slope of many of the dispersion curves. ${ }^{2}$ Because of the latter, the zoneboundary phonons of the high-temperature phase that become zone-center phonons in the low-temperature phase because of the cell doubling, may be degenerate with the zone-center phonons that are common to both phases. Consequently, in this work all observed lines other than the few leakage lines are assumed to be genuine and are classified accordingly.

In the ferroelectric phase, $\mathrm{Gd}_{2}\left(\mathrm{MOO}_{4}\right)_{3}$ is orthorhombic $\left(C_{2 V}\right)$ with 68 atoms/cell. All of the 201 optic phonons are Raman active. The Raman tensors and mode classifications for both phases of $\mathrm{Gd}_{2}\left(\mathrm{MoO}_{4}\right)_{3}$ are shown in Table I. It can be seen that there are $50 A_{1}, 51 A_{2}, 50 B_{1}$, and $50 B_{2}$ optic modes. Of these, 108 are internal modes, i.e., they are associated with coupled, internal vibrations of the $\mathrm{MoO}_{4}{ }^{--}$ions. The remaining $93 \mathrm{ex}-$ ternal modes are subdivided into $23 A_{1}, 24 A_{2}, 23 B_{1}$, and $23 B_{2} \cdot{ }^{6}$ Since the $A_{1}, B_{1}$, and $B_{2}$ symmetries are also ir active and $\mathrm{Gd}_{2}\left(\mathrm{MoO}_{4}\right)_{3}$ is noncentrosymmetric, special care must be taken in the selection of the Raman-scattering geometry to ensure that the phonon propagation is along a symmetry axis. If it is not, mode mixing due to polariton angular dispersion ${ }^{7}$ can occur and complicate the process of identification of the Raman-line symmetries.

\section{EXPERIMENTAL DETAILS}

Raman spectra were measured with a Spex model 1401 double monochromator, with about $1.0-\mathrm{cm}^{-1}$ 
spectral resolution. The excitation source was a Coherent Radiation Laboratory Model 52 argon-ion laser. Both the 4880 - and 5145- $\AA$ lines were used with input powers of $500 \mathrm{~mW}$. The signal was detected with a thermoelectrically-cooled EMI 9502 BA photomultiplier tube along with standard photon-counting electronics.

The samples were cut from a $c$-axis boule purchased from the Isomet Corporation. All samples were cut into cubes $6 \mathrm{~mm}$ on a side with their faces parallel to the orthorhombic crystallographic axes.

The samples were mounted on a cold finger in a conventional liquid-nitrogen optical Dewar. A nom- inal 300- $\Omega$ heater coil of enamel-coated 40 -gauge manganin wire was wrapped around a copper sample holder and thermally anchored with GE 7031 varnish. The temperature was measured with a resistance thermometer consisting of a $0.1-\mathrm{W}$ carbon resistor with a resistance of $4750 \Omega$ at $300^{\circ} \mathrm{K}$ connected to a Wheatstone bridge and null detector. Temperature variations of $0.5^{\circ} \mathrm{K}$ could be detected over the entire range.

\section{EXPERIMENTAL RESULTS}

The identifications of the Raman lines according to the point symmetries of the corresponding pho-

TABLE I. (a) Symmetry correlation of the low- and high-temperature phases [J. Petzelt and V. Dvorak, Phys. Status Solidi B 46, 413 (1971)]; (b) Raman tensors for the two phases [R. Loudon, Adv. Phys. 13, 423 (1964)].

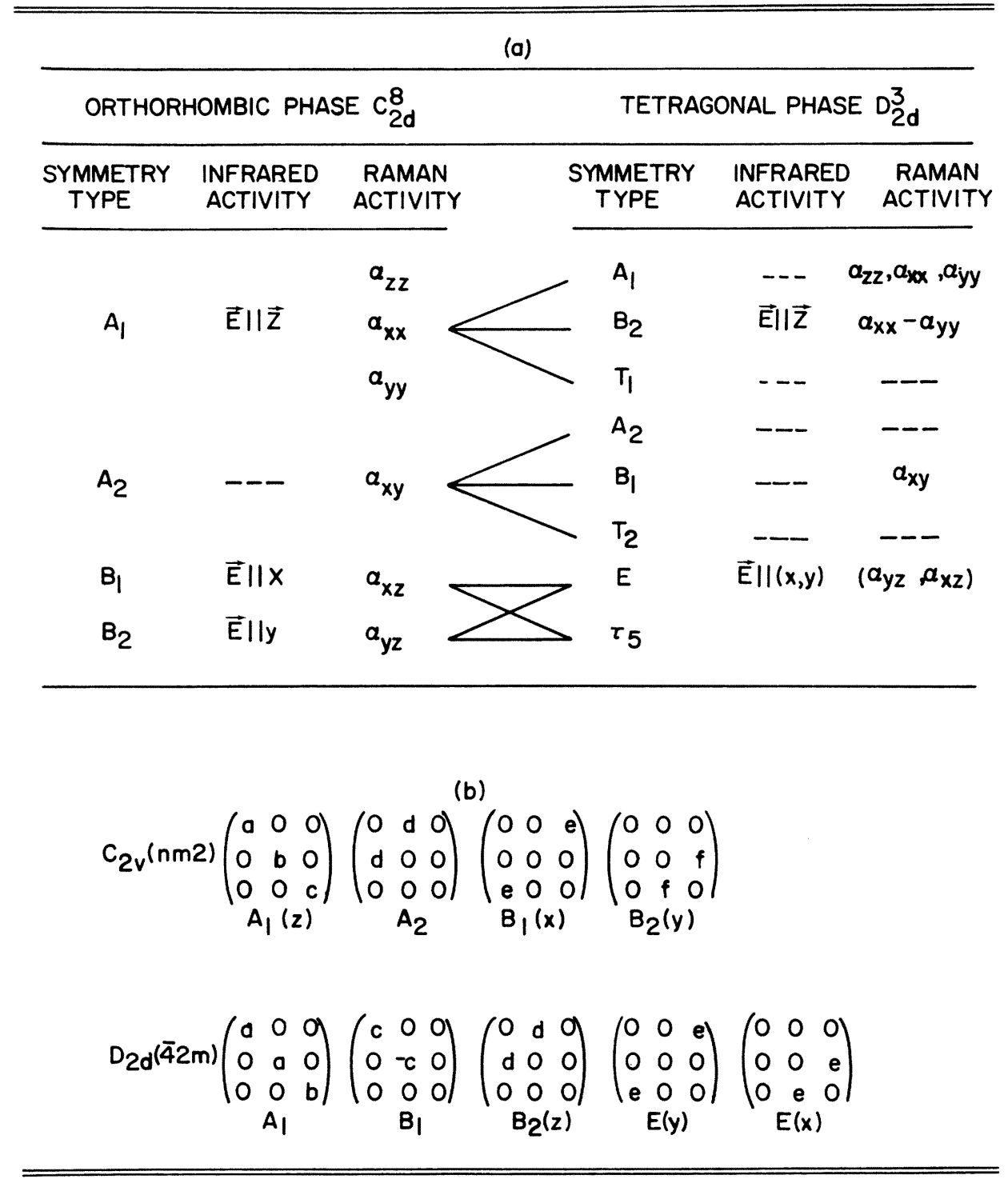


non modes can be made from inspection of the equation for the scattering efficiency ${ }^{8}$

$$
S=A\left(\epsilon_{\sigma} R_{\sigma \rho} \epsilon_{\rho}\right)^{2}, \quad \sigma, \rho=x, y, z,
$$

where $R_{\sigma \rho}$ is the appropriate Raman tensor for the crystal class, $\epsilon_{\sigma}$ and $\epsilon_{\rho}$ are the components of the unit vectors characterizing incident and scattered beam polarizations, respectively, and $A$ is a proportionality constant.

For infrared-active phonons in a biaxial crystal such as $\mathrm{Gd}_{2}\left(\mathrm{MoO}_{4}\right)_{3}$, this identification is not, in general, valid. Since the $A_{1}, B_{1}$, and $B_{2}$ modes are infrared active, the Raman spectrum for a given point symmetry will depend on the phonon propagation direction. To analyze such cases properly, the polariton dispersion relation should be used. For large-angle scattering, where the polariton velocity is small compared to the velocity of light in the medium, the polariton dispersion relation reduces to ${ }^{7}$

$$
\epsilon_{1} q_{1}^{2}+\epsilon_{2} q_{2}^{2}+\epsilon_{3} q_{3}^{2}=0
$$

where the $q_{i}$ are the components of the phonon

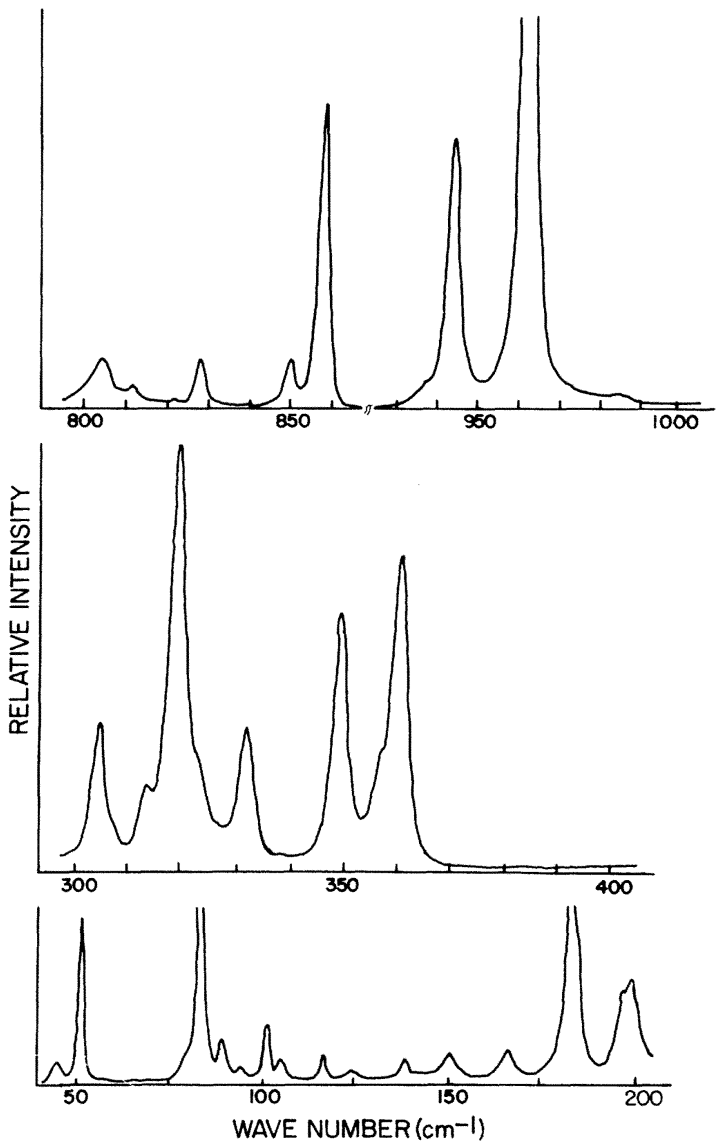

FIG. 1. $A_{1}(\mathrm{TO})$ spectra, $x(z z) y, 80^{\circ} \mathrm{K}$ (a weak line at $210 \mathrm{~cm}^{-1}$ is not shown). propagation vector and the $\epsilon_{i}$ are principal-axis dielectric functions, given by

$$
\epsilon_{i}=\epsilon_{1}^{\infty}+\sum_{j=1}^{50} \frac{s_{j}}{\omega_{0 j}^{2}-\omega^{2}}
$$

Here, $s_{j}$ is the oscillator strength and $\omega_{0 j}$ is the TO frequency of the $j$ th mode that contributes to the $i$-axis dielectric function.

The right-angle scattering geometries used in these measurements were $x(z z) y, x(y x) y, x(z y) z$, and $y(z x) z$ which from Eqs. (1)-(3) can be shown to correspond, respectively, to $A_{1}(z), A_{2}, B_{2}(y)$, and $B_{1}(x)$ symmetries. It was important in this work to obtain the $A_{1}(\mathrm{TO})$ and $A_{1}(\mathrm{LO})$ spectra separately.

TABLE II. Observed $A_{1}$ lines ( $T<T_{c}$ ) and corresponding lines of the high-temperature $\left(D_{2 d}\right)$ phase in $\mathrm{cm}^{-1}$ (sh, shoulder; bo, barely observable).

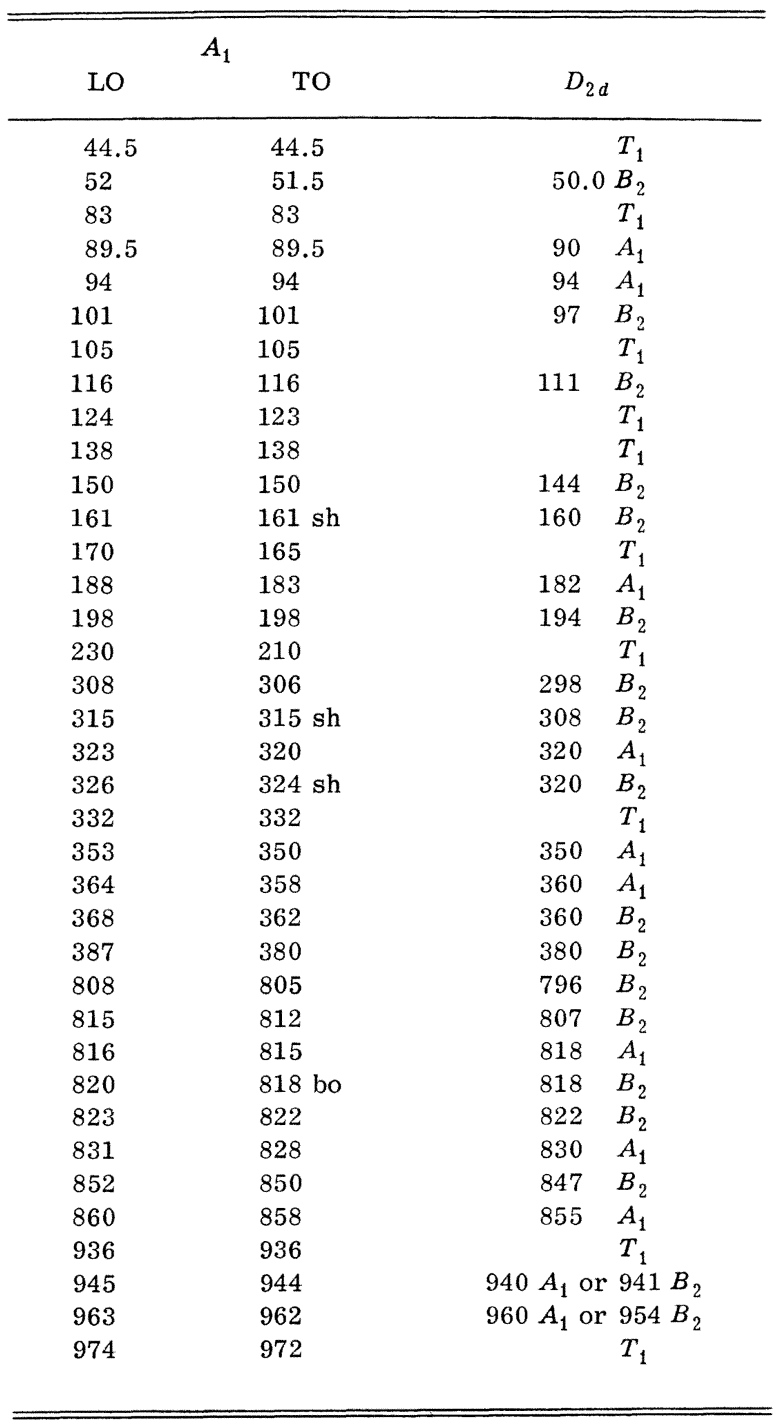


From Eq. (2), it can be seen that to obtain only an LO-phonon spectrum the phonon propagation direction must be along a principal axis. Consequently the $A_{1}(\mathrm{LO})$ spectrum was studied in the backscattering configuration $z(x x) \bar{z}$.

The Raman lines fall into two spectral groupings, $0-400$ and $740-1000 \mathrm{~cm}^{-1}$. From an examination of the figures and tables, discussed below, it can be seen that a few lines that can be seen in the figures have not been tabulated. We have included in the tables only those lines that can be identified unequivocally as part of the first order spectrum. (Identification of weak lines always involves some degree of uncertainty since they may be artifacts from electrical or optical noise, leakage from other symmetries, or of higher order o) In the $A_{1}$ spectra, we have applied the additional restriction that pairs of TO and LO lines had to appear reproducibly. The lines that can be seen at 119,181 , 298 , and $312 \mathrm{~cm}^{-1}$ and between 230 and $280 \mathrm{~cm}^{-1}$ did not satisfy this last criterion.

The $A_{1}(\mathrm{TO})$ and $A_{1}(\mathrm{LO})$ spectra $\left(\right.$ all at $80^{\circ} \mathrm{K}$ ) are

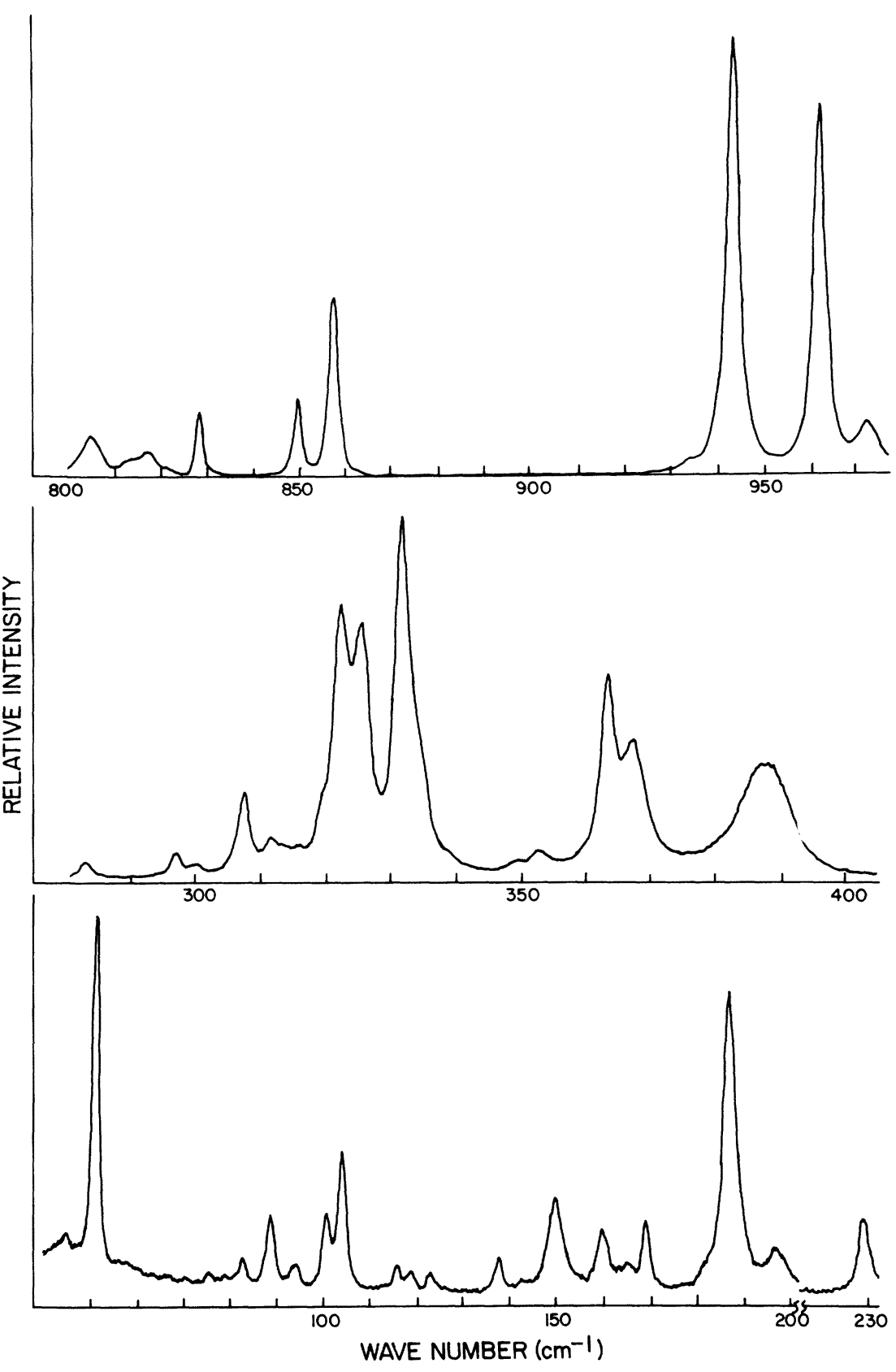

FIG. 2. $A_{1}$ (LO) spectra, $z(x x) \bar{z}, 80^{\circ} \mathrm{K}$. 
shown in Figs. 1 and 2, respectively, $B_{1}$ spectra in Fig. 3, $B_{2}$ spectra in Fig. 4 , and $A_{2}$ spectra in Fig. 5.

In Tables $I-V$, the frequencies of all the firstorder lines are listed along with their correlation with corresponding lines ${ }^{1}$ from the high-temperature phase. (Some of these high-temperature lines were excluded in our earlier work. ${ }^{1}$ ) Identification of the $D_{2 d}$ phonons that correlate with $C_{2 V}$ phonons of $A_{2}$ symmetry poses a special problem because $A_{2}$ modes are not Raman observable in the $D_{2 d}$ phase and, thus, whether or not the correlation is with an $\boldsymbol{A}_{2}$ or a zone-boundary phonon is not apparent. The polarizability changes associated with the zone-boundary, $T_{2}$ modes are likely to be small compared to those associated with the Raman-inactive $A_{2}$ modes. Therefore, strong $A_{2}$ lines are identified here as $A_{2}$ in the $D_{2 d}$ phase; otherwise, they are listed as either " $A_{2}$ or $T_{2}$."

An $A_{1}$ (TO) line, $47 \mathrm{~cm}^{-1}$ at room temperature,

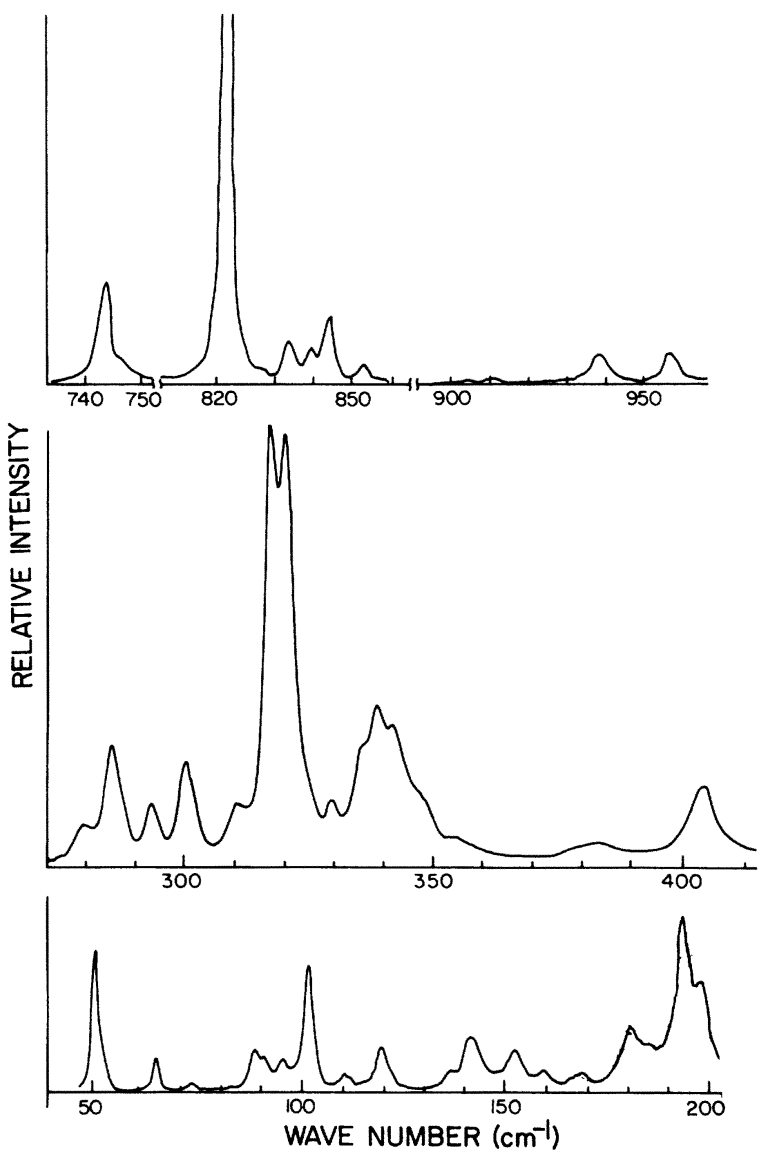

FIG. 3. $B_{1}$ spectra, $x(z y) z, 80^{\circ} \mathrm{K}$.
TABLE III. Observed $B_{1}$ lines $\left(T<T_{c}\right)$ and corresponding lines of the high-temperature $\left(D_{2 d}\right)$ phase in $\mathrm{cm}^{-1}$.

\begin{tabular}{rrrr}
\hline \hline \multicolumn{1}{c}{$B_{1}$} & $D_{2 d}$ & $B_{1}$ & \multicolumn{1}{c}{$D_{2 d}$} \\
\hline 43.0 & $\tau_{5}$ & 328.0 & $\tau_{5}$ \\
50.5 & $50.0 E$ & 335.0 & $\tau_{5}$ \\
64.0 & $\tau_{5}$ & 341.0 & $341.0 E$ \\
91.0 & $90.0 E$ & 382.0 & $380.0 E$ \\
98.0 & $\tau_{5}$ & 404.0 & $404.0 E$ \\
112.0 & $\tau_{5}$ & 740.0 & $742.0 E$ \\
136.0 & $130.0 E$ & 745.0 & $\tau_{5}$ \\
141.5 & $E$ & 822.0 & $817.0 E$ \\
169.5 & $160.0 E$ & 838.0 & $\tau_{5}$ \\
187.0 & $E$ & 844.0 & $835.0 E$ \\
200.0 & $190.0 E$ & 848 & $844.0 E$ \\
285.0 & $279.0 E$ & 857.0 & $\tau_{5}$ \\
292.0 & $\tau_{5}$ & 911.0 & $915.0 E$ \\
300.0 & $\tau_{5}$ & 939.0 & $930.0 E$ \\
309.0 & $306.0 E$ & 957.0 & $965.0 E$ \\
320.0 & $325.0 E$ & & \\
\hline \hline
\end{tabular}

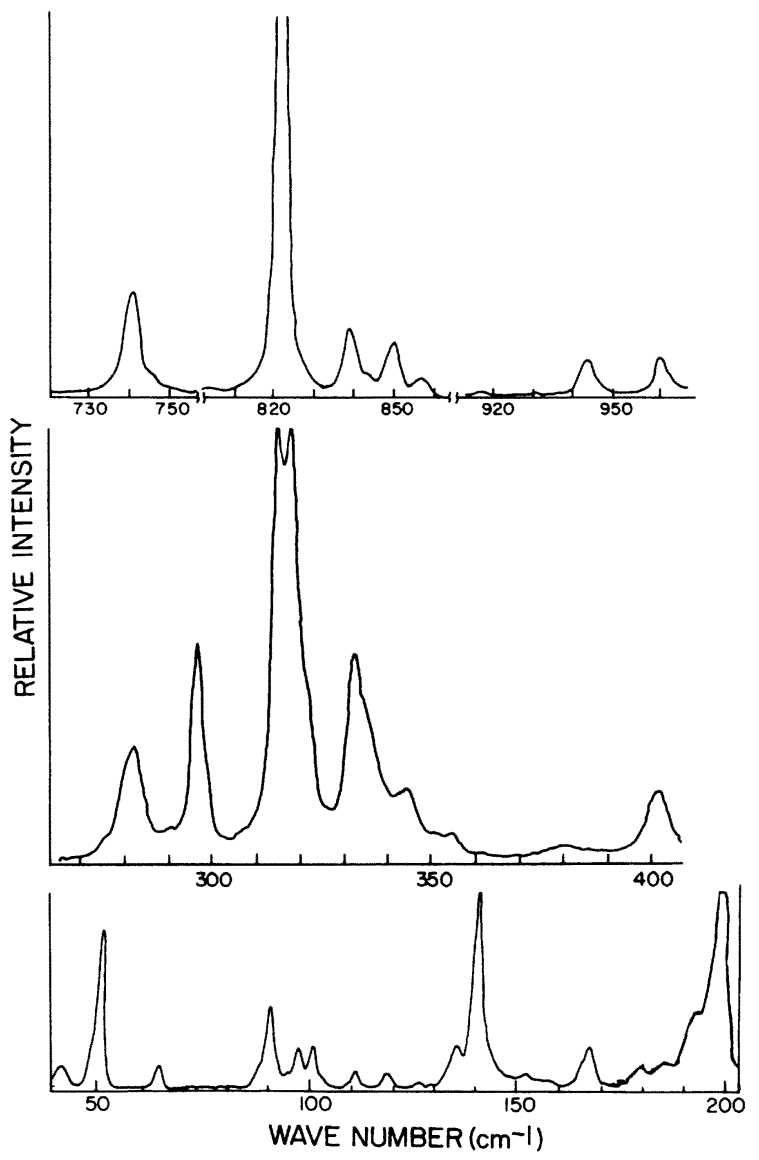

FIG. 4. $B_{2}$ spectra, $y(z x) z, 80^{\circ} \mathrm{K}$. 
whose peak frequency decreases on heating has been observed previously in $\mathrm{Gd}_{2}\left(\mathrm{MoO}_{4}\right)_{3} .^{3}$ Also, two overlapping $A_{1}$ (TO) modes have been observed in infrared absorption ${ }^{5}$ at low temperatures which coalesce into one broad line at room temperature, peaking at about $47 \mathrm{~cm}^{-1}$. Both the temperature $\mathrm{e}^{3}$ and stress $^{4}$ dependence above room temperature of this anomalous line are not exactly reproduced by a simple Lorentzian line.

We have reported briefly on the $A_{1}$ spectrum below $100 \mathrm{~cm}^{-1}$ previously. ${ }^{9} A_{1}$ (TO) spectra up to $100 \mathrm{~cm}^{-1}$ for four different temperatures between 80 and $300{ }^{\circ} \mathrm{K}$ are shown in Fig. 6. It can be seen that the $51.5-\mathrm{cm}^{-1}$ peak frequency decreases with increasing temperatures while its linewidth increases. This line has coalesced with the 44.5$\mathrm{cm}^{-1}$ line at room temperature, just as observed in infrared absorption. ${ }^{7}$ The peak frequency of the $44.5-\mathrm{cm}^{-1}$ line did not shift up to the highest temperature at which this line could still be resolved.

The peak frequency of the $83-\mathrm{cm}^{-1}$ line also decreased with increasing temperature to a value of

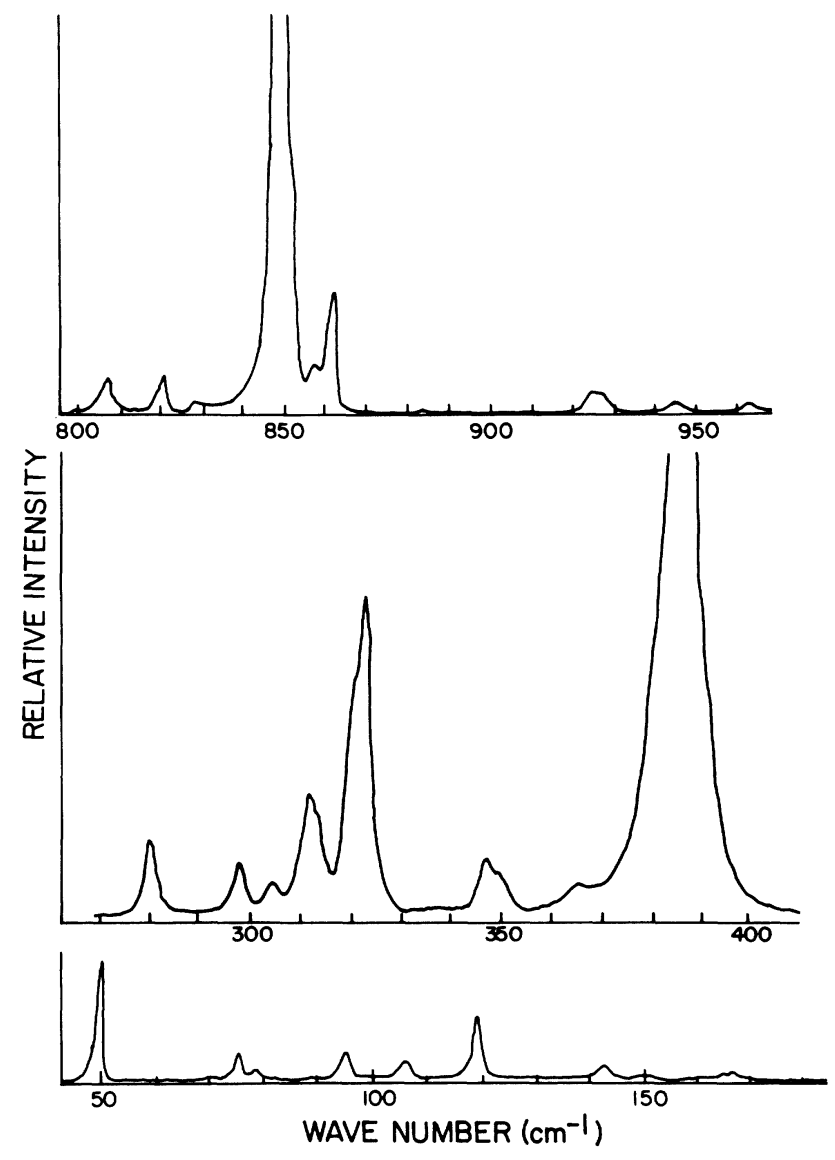

FIG. 5. $A_{2}$ spectra, $x(y x) y, 80^{\circ} \mathrm{K}$.
TABLE IV. Observed $B_{2}$ lines $\left(T<T_{c}\right)$ and corresponding lines of the high-temperature $\left(D_{2 d}\right)$ phase in $\mathrm{cm}^{-1}$.

\begin{tabular}{rrrr}
\hline \hline \multicolumn{1}{c}{$B_{2}$} & $D_{2 d}$ & $B_{2}$ & $D_{2 d}$ \\
\hline 49.0 & $50.0 E$ & 332.0 & $341.0 E$ \\
64.0 & $\tau_{5}$ & 334.0 & $\tau_{5}$ \\
95.0 & $\tau_{5}$ & 354.0 & $\tau_{5}$ \\
101.0 & $90.0 E$ & 379.0 & $380.0 E$ \\
110.0 & $\tau_{5}$ & 402.0 & $404.0 E$ \\
120.0 & $130.0 E$ & 740.0 & $742.0 E$ \\
152.0 & $E$ & 745.0 & $\tau_{5}$ \\
159.0 & $160.0 E$ & 820.0 & $817.0 E$ \\
180.0 & $E$ & 840.0 & $835.0 E$ \\
194.0 & $190.0 E$ & 844.0 & $\tau_{5}$ \\
272.0 & $279.0 E$ & 851.0 & $844.0 E$ \\
290.0 & $\tau_{5}$ & 857.0 & $\tau_{5}$ \\
296.0 & $306.0 E$ & 916.0 & $915.0 E$ \\
315.0 & $325.0 E$ & 944.0 & $930.0 E$ \\
320.0 & $\tau_{5}$ & 962.0 & $965.0 E$ \\
\hline \hline
\end{tabular}

$75 \mathrm{~cm}^{-1}$ at room temperature and remains at this value on heating up to the transition where it be comes Raman inactive (zone boundary mode), as shown in Fig. 7 (taken from Ref. 10).

$A_{1}$ (LO) spectra up to $100 \mathrm{~cm}^{-1}$, taken in the $z(x x) \bar{z}$ configuration, are shown in Fig. 8 for several temperatures from 80 up to $425^{\circ} \mathrm{K}$. [ Spectra for above room temperature were measured by one of us (BNG) in the UCLA Chemistry laboratories.] As in the TO spectra, the $44.5-\mathrm{cm}^{-1}$ line is obscured above room temperature by the broadening of the $51.5-\mathrm{cm}^{-1}$ line. However, the damping is much weaker in LO than in TO; the $51.5-\mathrm{cm}^{-1}$ line does not damp into the background below $T_{c}$ as in the TO spectrum. In fact, the intensity of the 51.5$\mathrm{cm}^{-1}$ line increases with temperature whereas the

TABLE V. Observed $A_{2}$ lines ( $T<T_{c}$ ), and corresponding lines of the high-temperature $\left(D_{2 d}\right)$ phase in $\mathrm{cm}^{-1}$.

\begin{tabular}{|c|c|c|c|}
\hline$A_{2}$ & $D_{2 d}$ & $A_{2}$ & $D_{2 d}$ \\
\hline 51 & $B_{1}$ & 314.0 & $T_{2}$ \\
\hline 76 & $A_{2}$ or $T_{2}$ & 322 & $T_{2}$ \\
\hline 79.0 & $A_{2}$ or $T_{2}$ & 323 & $326.0 B_{1}$ \\
\hline 95.0 & $94.0 \quad B_{1}$ & 348 & $A_{2}$ \\
\hline 106.0 & $A_{2}$ or $T_{2}$ & 350 & $T_{2}$ \\
\hline 119.0 & $114.0 \quad B_{1}$ & 366 & $T_{2}$ \\
\hline 142.5 & $144.0 \quad B_{1}$ & 387 & $385.0 B_{1}$ \\
\hline 70.5 & & 809 & $A_{2}$ \\
\hline 150.0 & $A_{2}$ or $T_{2}$ & 822 & $A_{2}$ \\
\hline 166.0 & $162.0 \quad B_{1}$ & 830 & $T_{2}$ \\
\hline 281 & $286.0 \quad B_{1}$ & 850 & $851.0 B_{1}$ \\
\hline 299 & $A_{2}$ & 858 & $T_{2}$ \\
\hline 305 & $T_{2}$ & 926 & $921.0 B_{1}$ \\
\hline 312.0 & 318.0 & 929 & $T_{2}$ \\
\hline
\end{tabular}


intensities of others such as the $83-\mathrm{cm}^{-1}$ line $(75$ $\mathrm{cm}^{-1}$ above $300^{\circ} \mathrm{K}$ ) decrease as the transition is approached. There is another important difference between these $A_{1}$ (TO) and $A_{1}$ (LO) spectra; the for mer remain $A_{1}$ above $T_{c}$ since the " $z z$ " polarizations are retained whereas the latter go from " $x x$ ", to " $x y, x y$," which corresponds to $A_{1}+B_{1}+B_{2}$ symmetry. From Table I, it can be seen that $A_{1}$ phonons of the low-temperature phase transform to $A_{1}, B_{2}$, or $T_{1}$ phonons in the high-temperature phase. There are no lines near $50 \mathrm{~cm}^{-1}$ in the $A_{1}$ spectrum above $T_{c}$. There is ${ }^{1}$ a strong $B_{2}$ line at $50 \mathrm{~cm}^{-1}$, and since according to Table I, $B_{2}$ modes above $\boldsymbol{T}_{c}$ must have corresponding $A_{1}$ modes but no corresponding modes of other symmetries below $T_{c}$, the $51.5-\mathrm{cm}^{-1}$ line must be a zone-center mode in both phases, going from $A_{1}$ to $B_{2}$ on heating through $T_{c}$. The $44.5-$ and $83-\mathrm{cm}^{-1}$ lines do not appear in either $A_{1}$ or $B_{2}$ spectra above $T_{c}$ and, therefore, must become $T_{1}$ zone-boundary modes.

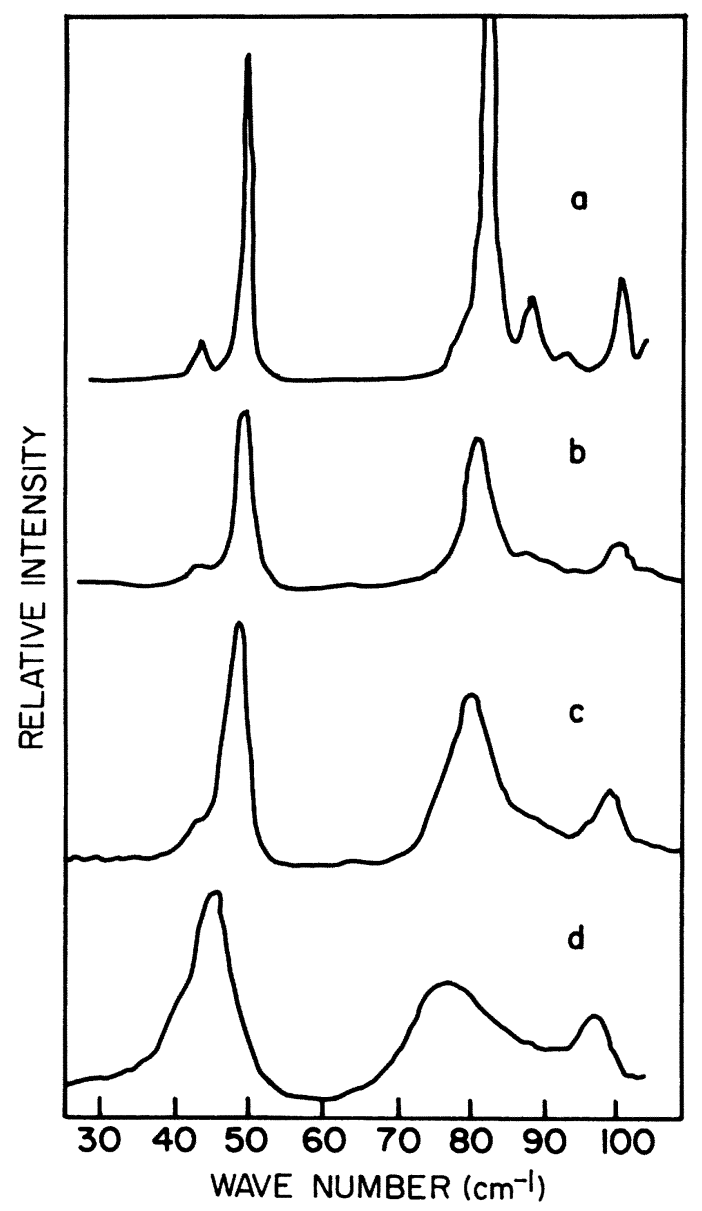

FIG. 6. $A_{1}$ (TO) spectra, $0-100 \mathrm{~cm}^{-1}$, for four temperatures: (a) $80^{\circ} \mathrm{K}$, (b) $160^{\circ} \mathrm{K}$, (c) $210^{\circ} \mathrm{K}$, and (d) $300^{\circ} \mathrm{K}$.
These, then, are the likely candidates for the two $A_{1}$ lines below $T_{c}$ that correspond to the two soft degenerate zone-boundary modes above $T_{c}$, as we have suggested previously. ${ }^{9}$

\section{DISCUSSION OF RESULTS}

By cooling to $80{ }^{\circ} \mathrm{K}$, it has been possible to resolve 126 of the possible 201 Raman-active modes. Among these, the behavior of the $A_{1}$ spectrum below $200 \mathrm{~cm}^{-1}$ is of most interest since it contains the only spectral features exhibiting abnormal temperature dependence. The ferroelectric phase transition has been shown ${ }^{11}$ to originate from the softening of two degenerate zone-boundary phonons on cooling to $T_{c}$. Below $T_{c}$, this degeneracy is removed and the two soft phonons become Ramanactive $A_{1}$ modes. These have not been identified unequivocally; in fact, the behavior below $T_{c}$ has not yet been elucidated. The explanation of the lattice dynamics of the ferroelectric phase will have to account for the anomalous damping of certain modes, ${ }^{3,4,9}$ the absence of a dielectric anoma$1 y,{ }^{12}$ the existence of anomalies in some of the elastic constants, ${ }^{13}$ and the ferroelasticity. ${ }^{14}$

If the Lyddane-Sachs-Teller relation is valid for this system, the observed absence of any dielectric anomaly for the clamped crystal must mean that the ratio of the LO to TO frequencies remains fixed, and if there is any softening, the two soften

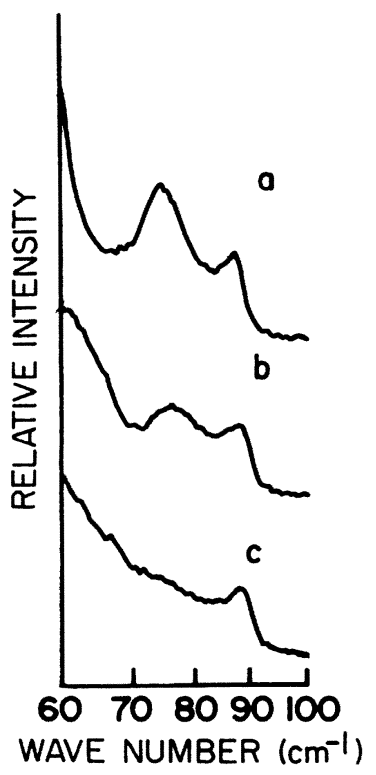

FIG. 7. Behavior of $83-\mathrm{cm}^{-1}$ line $\left(75 \mathrm{~cm}^{-1}\right.$ at $\left.300^{\circ} \mathrm{K}\right)$ on heating through $\boldsymbol{T}_{c}$ : (a) $300^{\circ} \mathrm{K}$, (b) $373^{\circ} \mathrm{K}<T<432^{\circ} \mathrm{K}$ $=T_{c}$, (c) $T>432^{\circ} \mathrm{K}$. 


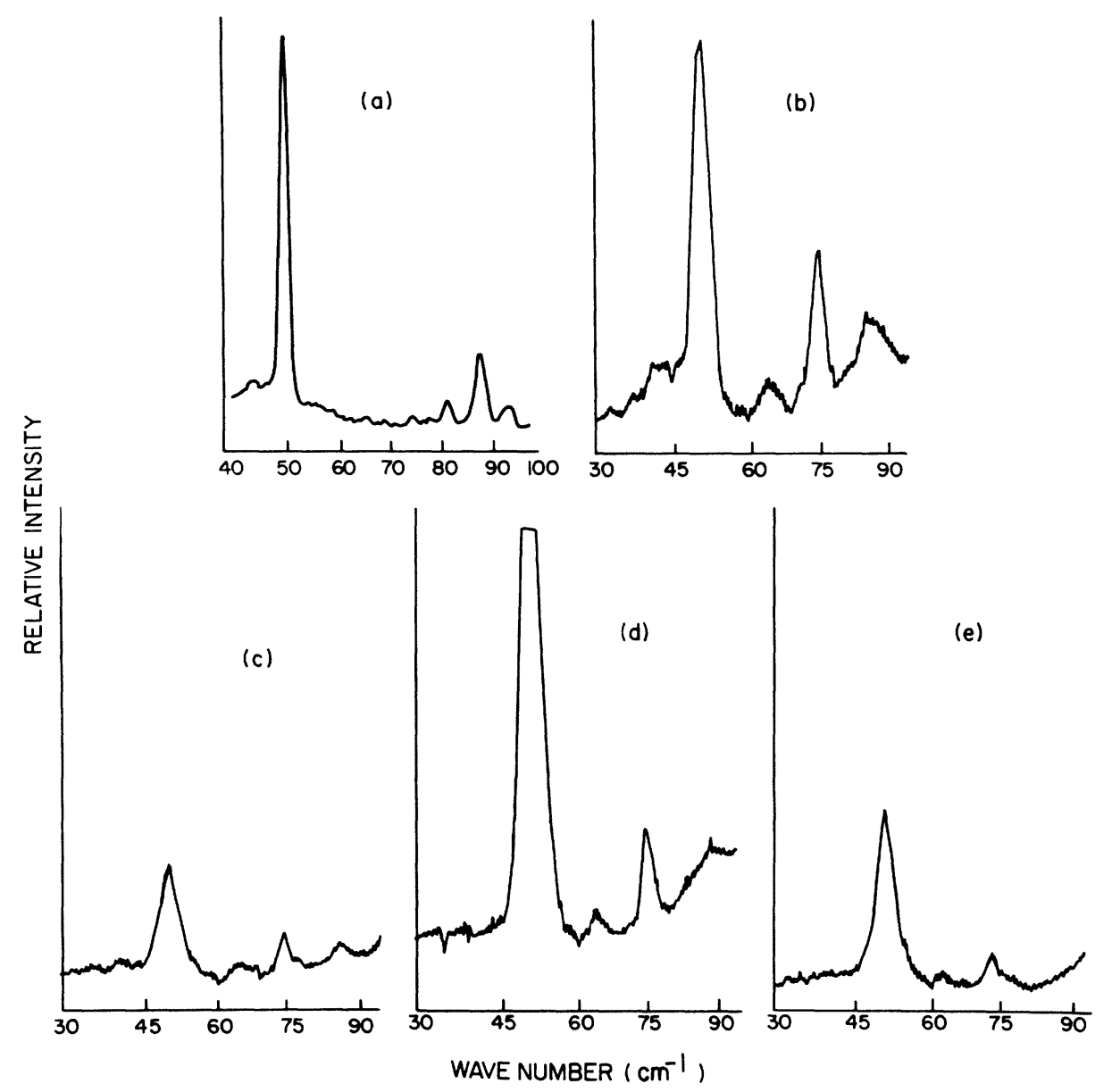

FIG. 8. $A_{1}(\mathrm{LO})$ spectra at various temperatures. (a) $80^{\circ} \mathrm{K}$, (b) $300^{\circ} \mathrm{K}$, (c) $363^{\circ} \mathrm{K}$, (d) $403^{\circ} \mathrm{K}$, and (e) $425^{\circ} \mathrm{K}$. together but the TO branch never reaches zero in the temperature range $T \leqslant T_{c}$. Dorner et al..$^{11}$ have suggested this type of behavior among several possible types for the two $A_{1}$ modes involved in the ferroelectric transition. This assumption of little or no softening, as observed, ${ }^{3,4,9}$ is consistent with the absence of the dielectric anomaly but offers no clue to the observed anomalies in the elastic constants. There is a clue, however, in the anomalous damping of the three coupled modes at $44.5,51.5$, and $83 \mathrm{~cm}^{-1}$ (at $\left.80^{\circ} \mathrm{K}\right)$. We have offered a brief explanation of this damping previously; it goes as follows. The $51.5-\mathrm{cm}^{-1} A_{1}$ (TO) mode that exhibits anomalous damping has been shown from measurements of the LO-phonon scattering to become a $B_{2}$ mode above $T_{c}$ and not a zone-boundary mode; therefore it cannot be one of the modes involved in the transition. From comparisons of the $80^{\circ} \mathrm{K}$ spectra with higher-temperature spectra, the $83-\mathrm{cm}^{-1}$ peak can clearly be seen to begin to soften on heating with a large amount of intensity transfer, presumably into the $51.5-\mathrm{cm}^{-1}$ peak, as evidenced by the much more rapid decrease in peak height of the $83-\mathrm{cm}^{-1}$ peak relative to the $51.5-$ $\mathrm{cm}^{-1}$ peak. Since the $51.5-\mathrm{cm}^{-1}$ mode is not soft ening, it "pins" the upper peak at $75 \mathrm{~cm}^{-1}$ above room temperature. The behavior of the $44.5-\mathrm{cm}^{-1}$ mode is not apparent above room temperature since it is obscured by the $51.5-\mathrm{cm}^{-1}$ mode. However, there is no indication that it softens. Since the damping of the $51.5-\mathrm{cm}^{-1}$ mode disappears above $T_{c}$, the damping mechanism may be associated with a nest of modes of small wave vector in the vicinity of $44 \mathrm{~cm}^{-1}$, of which the $44.5-\mathrm{cm}^{-1}$ mode is the zero wave-vector limit. We suggest that the damping is due to a two-phonon decay process involving one of these $44-\mathrm{cm}^{-1}$ modes and a long-wave acoustic phonon $\left(\sim 5-7 \mathrm{~cm}^{-1}\right)$. This implies a strong peak in the imaginary part of the self-energies of both the $50-\mathrm{cm}^{-1}$ phonon and the acoustic phonon. This, in turn, implies a marked negative shift in the real part of the self-energy for acoustic phonons of lower frequency $(<5-7$ $\mathrm{cm}^{-1}$ ). Thus there will be an anomalous decrease in the associated elastic constant. If no phase transition took place at $T_{c}$, the damping and de- 
crease in the elastic constant would continue to change with heating. However, the first-order change at $T_{c}$ in which the $44-\mathrm{cm}^{-1}$ modes move to the zone boundary causes the damping to disappear, and hence an apparent anomaly in the elastic constant. The elastic behavior is thus a consequence of the phase transition rather than one of its causes. There will also be an associated effect on the real part of the self-energy of the $50-\mathrm{cm}^{-1}$ phonon which could explain the failure of the simple Lorentzian fit. ${ }^{3} 4$ Specifically, the deviation between the measured and calculated spectra is such that the former is always higher than the latter be- low the peak and lower above. This is what is expected when the imaginary part of the self-energy shows a strong peak. ${ }^{15}$ In these circumstances, the real part will tend to decrease the effective frequency of the Lorentzian below the peak and increase it above. Such an effect would be in qualitative agreement with the observed behavior.

As mentioned above, the absence of any mode softening in the ferroelectric phase is consistent with some of the qualitative temperature dependences of the "soft modes" below $T_{c}$ suggested by Dorner, Axe, and Shirane ${ }^{11}$ and, therefore, should not be regarded as anomalous.
${ }^{\dagger}$ Research sponsored by the Air Force Office of Scientific Research, Office of Aerospace Research, USAF under Grant No. AFOSR 70-1926.

*This work is part of a dissertation submitted by B. N. Ganguly in partial fulfillment of the requirements for the Ph.D. degree.

¥ Present address: Dept. of Chemistry, University of California, Los Angeles, Calif. 90024.

${ }^{1}$ F. G. Ullman, B. J. Holden, B. N. Ganguly, and J. R. Hardy, Phys. Rev. B 8, 2991 (1973).

${ }^{2}$ L. L. Boyer and J. R. Hardy, Phys. Rev. B $\underline{8}, 2205$ (1973).

${ }^{3}$ P. A. Fleury, Solid State Commun. $\underline{8}, 601$ (1970).

${ }^{4}$ B. N. Ganguly, F. G. Ullman, J. R. Hardy, and R. D. Kirby, Phys. Rev. B 12, 3783 (1975).

${ }^{5}$ J. Petzelt, Solid State Commun. 9, 1485 (1971).

${ }^{6} \mathrm{~J}$. Petzelt and V. Dvorak, Phys. Status Solidi B $\underline{46}$,
413 (1971).

${ }^{7}$ A. S. Barker and R. Loudon, Rev. Mod. Phys. 44, 18 (1972); E. Anda, Solid State Commun. 9, 1545 (1971).

${ }^{8}$ R. Loudon, Adv. Phys. 13, 423 (1964).

${ }^{9}$ B. N. Ganguly, F. G. Ullman, R. D. Kirby, and J. R. Hardy, Solid State Commun. 17, 533 (1975).

${ }^{10}$ B. J. Holden, Ph.D. dissertation (University of Nebraska, 1971) (unpublished).

${ }^{11}$ B. Dorner, J. D. Axe, and G. Shirane, Phys. Rev. B $\underline{6}$, 1950 (1972).

${ }^{12}$ L. E. Cross, A. Fouskova, and S. E. Cummins, Phys. Rev. Lett. 21, 812 (1968).

${ }^{13}$ U. T. Hochli, Phys. Rev. B $\underline{6}, 1816$ (1972).

${ }^{14}$ K. Aizu, J. Phys. Soc. Jpn. 27,387 (1969).

${ }^{15}$ R. A. Cowley, Phonons (Oliver and Boyd, London, 1966), p. 170 . 